\title{
Robustness of topological corner modes in photonic crystals
}

\author{
Matthew Proctor $\odot,{ }^{1}$ Paloma Arroyo Huidobro $\odot,{ }^{2, *}$ Barry Bradlyn $\odot,{ }^{3, \dagger}$ María Blanco de Paz $\odot,{ }^{4}$ Maia G. Vergniory $\odot, 4,5$

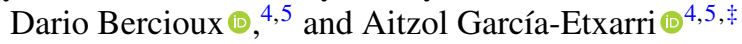 \\ ${ }^{1}$ Department of Mathematics, Imperial College London, London SW7 2AZ, United Kingdom \\ ${ }^{2}$ Instituto de Telecomunicações, Instituto Superior Tecnico-University of Lisbon, Avenida Rovisco Pais 1, Lisboa, $1049-001$ Portugal \\ ${ }^{3}$ Department of Physics and Institute for Condensed Matter Theory, University of Illinois at Urbana-Champaign, \\ Urbana, Illinois 61801-3080, USA \\ ${ }^{4}$ Donostia International Physics Center, 20018 Donostia-San Sebastián, Spain \\ ${ }^{5}$ IKERBASQUE, Basque Foundation for Science, Maria Diaz de Haro 3, 48013 Bilbao, Spain
}

(Received 20 July 2020; revised 17 September 2020; accepted 22 October 2020; published 9 December 2020)

\begin{abstract}
We analyze the robustness of corner modes in topological photonic crystals, taking a $C_{6}$-symmetric breathing honeycomb photonic crystal as an example. First, we employ topological quantum chemistry and Wilson loop calculations to demonstrate that the topological properties of the bulk crystal stem from an obstructed atomic limit phase. We then characterize the topological corner modes emerging within the gapped edge modes employing a semianalytical model, determining the appropriate real-space topological invariants. We provide a detailed account of the effect of long-range interactions on the topological modes in photonic crystals, and we quantify their robustness to perturbations. We conclude that, while photonic long-range interactions inevitably break chiral symmetry, the system is reducible to a chirally symmetric limit and the corner modes are protected by this together with lattice symmetries.
\end{abstract}

DOI: 10.1103/PhysRevResearch.2.042038

Introduction. Photonic topological insulators host protected boundary modes that are robust against a range of defects and imperfections [1]. While the paradigmatic case of two-dimensional (2D) topological photonic crystals (PhCs) hosting one-dimensional (1D) edge modes immune to backscattering has been extensively studied [2], a hierarchy of protected boundary states of lower dimensionality are possible in higher-order topological insulators (HOTIs) [3]. For instance, quantized quadrupole insulators in $2 \mathrm{D}$, which were introduced in a generalization of the Su-Schrieffer-Heeger (SSH) model to a square lattice with a flux [3], host 1D edge states, as well as zero-dimensional (OD) corner modes. These higher-order topological modes (HOTMs) localized at the OD corners of a 2D lattice benefit from topological protection. Just as HOTIs in condensed matter systems are characterized by charge fractionalization due to a filling anomaly of the bulk states [4-7], classical wave HOTIs reveal an analogous fractional corner anomaly of the density of states [8]. In systems with short-range hoppings and approximate chiral symmetry, these corner modes are midgap states $[9,10]$.

\footnotetext{
*p.arroyo-huidobro@1x.it.pt

†bbradlyn@illinois.edu

*aitzolgarcia@dipc.org
}

Published by the American Physical Society under the terms of the Creative Commons Attribution 4.0 International license. Further distribution of this work must maintain attribution to the author(s) and the published article's title, journal citation, and DOI.
HOTMs have been realized in a variety of classical systems [11-25], and their robustness has been exploited for stable lasing [26-28]. However, a rigorous study of the effect of long-range interactions (LRIs) which is unavoidable in many photonic systems [15,29-33], as well as a detailed analysis of the robustness of the HOTMs has not been undertaken. Notice that in this Rapid Communication "interactions" refer to the photonic coupling between different sites. Here we consider a $\mathrm{PhC}$ with a $C_{6}$-symmetric lattice $[16,34,35]$, and fill the aforementioned gap by taking advantage of a semianalytical model with LRIs [36], that is, interactions beyond nearest neighbors between all the lattice elements. This allows us to perform an extensive study of the robustness of these modes against defects and imperfections. Crucially, we show that the HOTMs are protected by lattice symmetries; we quantify their degree of robustness against chiral symmetry breaking LRIs, as well as to strong defects. With our analysis, we clarify some misconceptions about the topological origin of corner states in $C_{6}$-symmetric lattices [35,37].

Photonic crystal. We consider the breathing honeycomb $\mathrm{PhC}$ in Fig. 1(a). Each unit cell in the triangular lattice consists of six silicon rods $(\varepsilon=11.7)$ in vacuum of radius $r=0.12 a_{0}$ located at a distance $R=R_{0}(1 \pm \delta)$ from the origin of the unit cell. Here, $a_{0}$ is the lattice parameter, and $R_{0}=a_{0} / 3$ the location of the rods in the unperturbed honeycomb arrangement. The perturbation of the honeycomb lattice of rods by $\pm \delta$ yields expanded and contracted phases, respectively, where the doubly degenerate Dirac point at $\Gamma$ splits and a bulk band gap opens between $\omega a /(2 \pi c)=0.4-0.5$, Fig. 1(b). This band gap hosts $1 \mathrm{D}$ edge states as measured in several photonic 


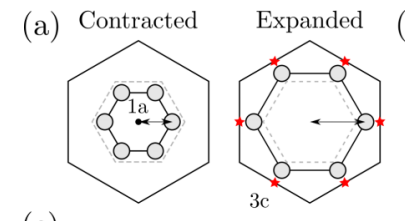

(c)

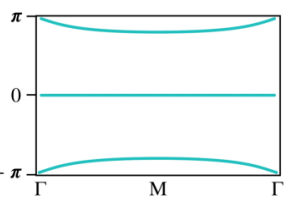

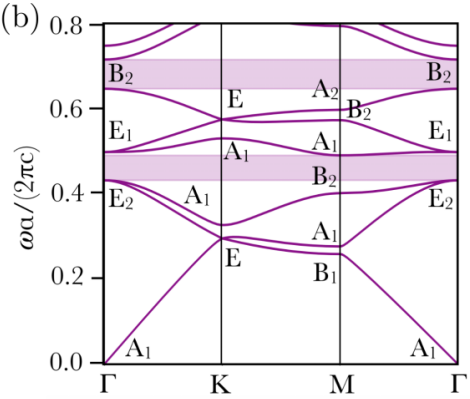

FIG. 1. (a) Unit cells of the bulk lattice in the contracted and expanded phases, characterized by a contraction/expansion parameter, $\delta$. The relevant Wyckoff positions are labeled 1a (black circle) and $3 \mathrm{c}$ (red star). (b) Band structure of the silicon photonic crystal in the expanded phase for the TM modes. The expansion coefficient $\delta$ is 0.11 , and the radius of the cylinders is $0.12 a_{0}, a_{0}$ being the lattice constant of the crystal. (c) Wilson loops for bands 4-6 (Wilson loops for bands 1-3 are similar [38]).

experiments [39-45]. We now discuss how they are not an instance of a $\mathbb{Z}_{2}$ topological insulator [46].

Figure 1(b) presents the band structure of the expanded phase $(\delta=0.11)$. We first determine the topological properties of the system through the application of topological quantum chemistry $[47,48]$. The irreducible representations of the eigenfields at the high symmetry points (irrep labels), displayed in the band structure, are calculated using GTPack $[49,50]$. Using the catalog of elementary band representations (EBRs) in the Bilbao Crystallographic Server [47,51-55], along with the irrep labels we can identify the topological properties of each set of connected bands. Counting from $\omega=0$, bands 4-6 are all interconnected and their irrep labels are accordant to Wannier functions centered in the $3 c$ Wyckoff position transforming in the $\left(E_{1} \uparrow G\right)_{3 c}$ band representation [56]. Since these bands can be identified with an EBR, we conclude that the system presents a trivial $\mathbb{Z}_{2}$ topological invariant. Nevertheless, the $3 c$ Wyckoff position of the band representation indicates that the Wannier functions of this set of bands are not centered around the origin of the unit cell, but at their edges. This can be understood as a 2D analog of the topological hybridization of eigenstates of a 1D SSH chain. This topological phase was labeled in the past in analogy with solid-state systems as the photonic obstructed atomic limit (OAL), because although an atomic limit exists, it is "obstructed" since the Wannier centers are not located at the position where the photonic "atoms" sit [57], the photonic atom being the collection of the six contracted/expanded cylinders inside the unit cell. Moreover, we characterize our system through the calculation of the eigenvalues of the Wilson loop [57] for this set of connected bands, Fig. 1(c). The resulting Wilson loops present no windings (characteristic of $\mathbb{Z}_{2}$ or Chern insulators), but the Wannier centers are not only localized in the origin of the unit cell $(W=0)$ as in a trivial system, but also at its edges $(W= \pm \pi)$, indicating that the system presents an obstruction similar to the 1D SSH chain [58]. On the other hand, the PhC in the contracted phase is a trivial photonic insulator. This can be seen from the Wannier centers of the EBRs being located at the origin of the unit cell (1a Wyckoff position), and by looking at the eigenvalues of the Wilson loop [38].

It should be emphasized here that in 2D systems, there is a subtle relationship between OAL and HOTIs. In toy models with nearest-neighbor interactions, provided there is an even number of elements in the unit cell, then a lattice is chirally symmetric [59]. Chiral symmetry, or sublattice symmetry, forces the energy spectrum to be symmetrical about a fixed value (often taken to be zero in the literature). If an OAL model has chiral symmetry, then it is sometimes possible to define a bulk topological invariant which counts the number of OD corner modes in a finite-sized system preserving the crystal symmetries. Systems with nonzero values of this invariant are properly termed HOTIs.

In the absence of chiral symmetry, there is no guarantee that a finite-sized system will have corner modes pinned to a special frequency. These systems are regarded as OAL systems and can be characterized by the centers of their Wannier functions (as above, and see Ref. [60]), by real-space invariants (see [38] and Refs. [61,62]) or by a filling anomaly (see [38] and Refs. [5,6]). The photonic finite-sized breathing honeycomb lattice breaks chiral symmetry due to unavoidable LRIs. However, for the remainder of this work we will exploit the fact that the system is deformable to a chiral-symmetric limit. This allows us to make semianalytical predictions about the presence and robustness of corner modes. We emphasize that this is possible because in the limit of short-range interactions, the system is chirally symmetric. In comparison, any lattice with an odd number of elements in the unit cell is incompatible with chiral symmetry, even in the limit of short-range interactions $[4,15,60,63]$.

While the 0D corner modes in 2D SSH-like PhC particles (finite-size crystals) with $C_{4}$ symmetry have been extensively explored [12-14,26,27], in PhC particles with $C_{6}$ symmetry, only the 1D edge states have been studied [64-66]. We analyze the emergence of $0 \mathrm{D}$ photonic corner states in this system by looking at 2D particles made of cells in the expanded phase and surrounded by cells in the contracted phase [see Fig. 2(a)]. With the chosen edge termination [Fig. 2(b)], the lattice sites along the interface are well dimerized, resulting in corner modes well isolated in frequency; in comparison to an interface with complete unit cells at the interface [16,35,37], see [38] for more details. Results of MIT Photonic Bands (MPB) supercell calculations [67] are shown in Figs. 2(c) and 2(d). The frequency eigenvalues 2(c) show a band gap with six midgap states. The real part of the displacement field eigenvectors $D_{z}$ for these six states are shown in Fig. 2(d). These are concentrated at the corners of the particle, thus we classify them as corner modes, marked in red in Fig. 2(c). States $2 \mathrm{~A}$ and $2 \mathrm{~B}$ and $3 \mathrm{~A}$ and $3 \mathrm{~B}$ are degenerate pairs. The states immediately above and below the band gap are edge states (cyan) [64-66], followed by bulk eigenstates (gray). Thus, the 0D corner states in this PhC particle are hosted within the gapped $1 \mathrm{D}$ edge states, in contrast to corner modes in $C_{3}$ - and $C_{4}$-symmetric PhCs [13-15].

Coupled dipole model. Since the spectrum of the PhC particle is determined by lattice symmetries together with LRIs, we now exploit a semianalytical model to unveil the properties of corner modes in a closely related nanophotonic system, an array of subwavelength-spaced dipolar nanoparticles. The 


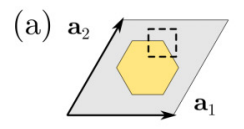

(b) sublattice: $\bullet a \bullet$

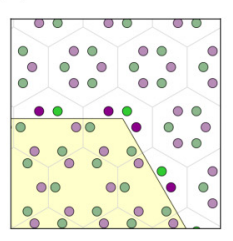

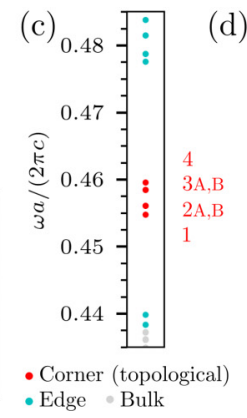

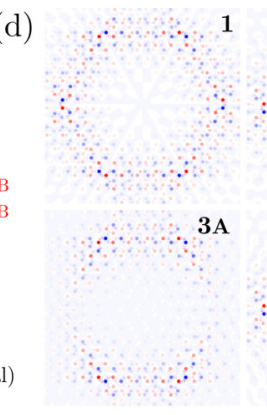

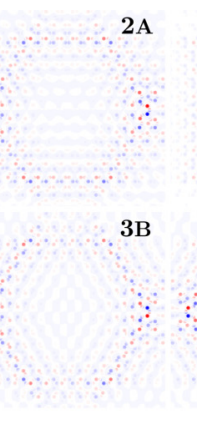

$2 \mathrm{~A}$

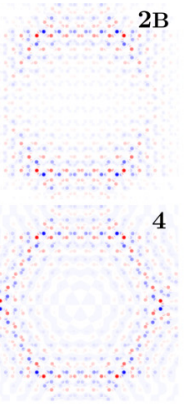

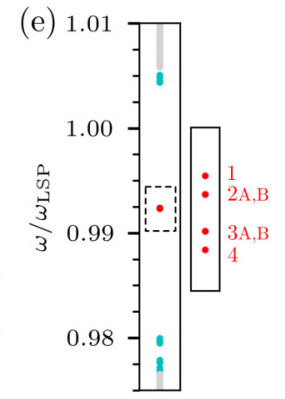

(f)

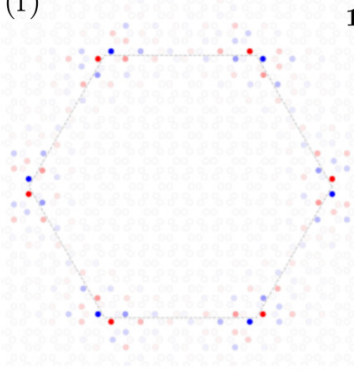

FIG. 2. (a) Scheme for the topological particle supercell. (b) Particle lattice, with sublattices $a$ (green) and $b$ (purple). (c), (d) Modes of a photonic crystal particle: Frequency $(\omega)$ of topological corner (red), edge (cyan), and bulk (gray) states (c), and displacement field plots, showing $D_{z}$ (d). (e), (f) Quasistatic model of the topological particle. (e) Frequency of topological corner, edge, and bulk states, for silver nanoparticles with radius $10 \mathrm{~nm}$ and height $40 \mathrm{~nm}$. (f) Dipole moments of the six corner eigenmodes. In the color scale used in (d) and (f) red (blue) represents positive (negative) values. In both cases, $\delta=0.11$.

coupled dipole model (CDM) is a versatile method for investigating the optical response of arrays of subwavelength elements such as cold atoms or plasmonic nanoparticles (NPs) [36]. Within this model we can reproduce all the relevant features found in full field simulations of the $\mathrm{PhC}$ topological particle. Then, we use it to shed further light on the properties of the corner modes, particularly on their robustness against disorder.

For a system of point dipoles (in the absence of an external electric field), a coupled dipole equation can be written to model the interaction between all dipoles. The electric dipole moment $\mathbf{p}$ at position $\mathbf{d}_{i}$ due to a dipole at $\mathbf{d}_{j}$ is

$$
\frac{1}{\alpha(\omega)} \mathbf{p}_{i}=\hat{\mathbf{G}}\left(\mathbf{d}_{i j}, \omega\right) \cdot \mathbf{p}_{j},
$$

with frequency $\omega$ and separation $\mathbf{d}_{i j}$. The polarizability $\alpha(\omega)$ describes the optical response of a single NP. The dipoledipole interactions are given by the dyadic Green's function $\hat{\mathbf{G}}$. For a periodic system, we can write the following system of an eigenvalue equation:

$$
\left[\hat{\mathbf{I}} \frac{1}{\alpha(\omega)}-\hat{\mathbf{H}}\left(\mathbf{k}_{\|}, \omega\right)\right] \cdot \mathbf{p}=0,
$$

with Bloch wave vector $\mathbf{k}_{\|}$. The interaction matrix $\hat{\mathbf{H}}$ contains the lattice sums [36] and this model goes beyond tightbinding, nearest-neighbor models by including interactions between all the lattice elements. The modes of the system can be found by solving the eigenvalue equation, and the resonance frequencies can then be extracted from the polarizability [38].

In Figs. 2(e) and 2(f) we present the results of the CDM for the OAL particle with the same geometry as the PhC. Here we particularize the system to the out-of-plane modes of subwavelength spheroidal metallic NPs, which correspond to the $\mathrm{TM}$ modes in the $\mathrm{PhC}$. We take a quasistatic approximation, and only include the near-field interaction term in the Green's function $\left(\propto 1 / d^{3}\right)$, which is accurate for these subwavelength NPs. Figure 2(e) shows the frequency spectrum around the band gap with corner modes within the gapped edge and bulk bands. For the plasmonic system, zero eigenvalue $(E=0)$ maps to $\omega_{\mathrm{LSP}}$, the localized surface plasmon frequency of the NPs. We see that the center of the band gap is located close to but not exactly at $\omega_{\mathrm{LSP}}$, and that the spectrum is not exactly symmetric around that point. This is a consequence of chiral symmetry breaking due to LRIs, as we discuss below.

In Fig. 2(f) we plot the dipole moments of the first midgap corner eigenmode, which reproduce well the $D_{z}$ field distributions of the $\mathrm{PhC}$. Importantly, the corner modes are localized on one sublattice, while the dipole moments in the opposite sublattice remain virtually zero [see Fig. 2(b)]. A similar sublattice localization of corner modes is present in the $\mathrm{PhC}$, though weaker due to the fully retarded interactions. Nevertheless, this shows that both systems are approximately chiral symmetric despite the LRIs, which has implications on the robustness of these $0 \mathrm{D}$ modes. In addition, these modes are well separated from the gapped bulk and edge states and are tightly confined to the corners. We now use the CDM to better characterize the properties of the corner modes. First, we study the behavior of the system as a function of $\delta$, the deviation of the lattice of NPs away from a honeycomb. In Fig. 3(a), we plot the eigenvalue spectrum, such that the symmetry properties of the spectrum around zero eigenvalue
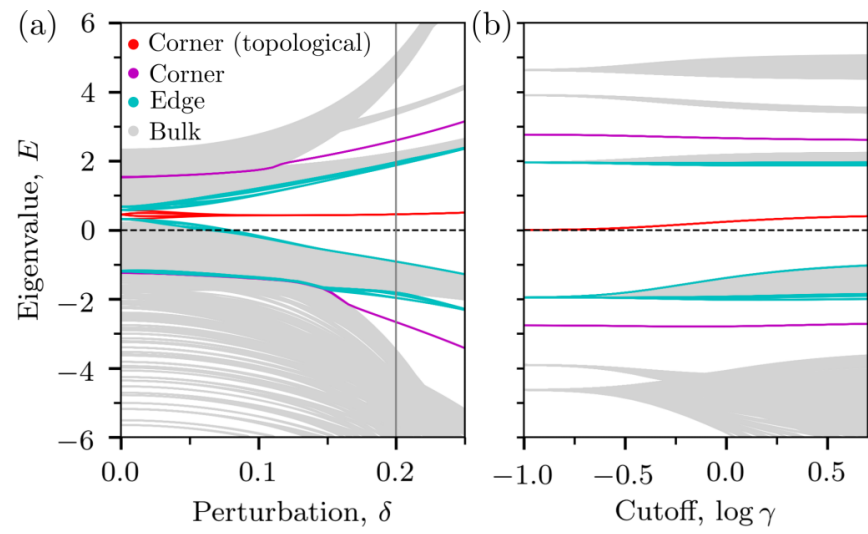

FIG. 3. Topological particle eigenvalues. (a) Evolution with increasing unit cell perturbation $\delta$, with topological corner modes (red) well separated from the edge (cyan) and bulk (gray) modes. Other corner modes are shown in magenta. (b) Dependence on interaction length between lattice sites, $\gamma$, for $\delta=0.2$. As interactions go from nearest neighbors $\gamma=0.1$ to long range, chiral symmetry is broken and the spectrum is no longer symmetrical about $E=0$. 
are clearer. Starting from the unperturbed honeycomb lattice $(\delta=0)$, we see how increasing $\delta$ controls the size of the bulk band gap. At the same time, the corner modes (red) stay at approximately constant eigenvalue, only slightly shifted away from zero due to the inherent breaking of chiral symmetry. Edge modes (cyan) appear at the edges of the bulk bands. As $\delta$ increases, the corner modes are more isolated in the band structure, and hence more strongly confined to corners of the particle. For $\delta \gtrsim 0.12$ new sets of corner modes (magenta) emerge from the bulk for positive and negative eigenvalues. In contrast to the corner modes discussed here, these modes do not lie at the middle of the gap, and they are not localized only on one of the sublattices.

The CDM also enables us to analyze the photonic corner modes analytically, as detailed in the Supplemental Material [38]. We find that when interactions are short range, the eigenvalue problem for the coupled dipoles maps onto a tight-binding Schrödinger equation for a system with six $s$ orbitals at the $6 d$ Wyckoff position in the unit cell (there is one $s$ orbital at the position of each NP). As $\delta$ increases, the model undergoes a transition between an atomic limit phase with Wannier centers on the $1 a$ position, to an OAL phase with Wannier centers on the $3 c$ position; in the shortrange limit these Wannier functions are compactly supported, and can be found exactly. For a finite-sized system, the two atomic limits are distinguished by the $p 6 \mathrm{~mm}$ real-space invariants of Ref. [61], which confirms that HOTMs are protected by lattice symmetries. Furthermore, we can solve for the corner modes in a topological particle in the long-wavelength approximation. We find that the low-energy theory of the domain between trivial and OAL particles naively resembles the edge of a quantum spin Hall (QSH) insulator if only the lowest-order terms are considered. However, when we include crystalline- and chiral-symmetric perturbations, we find that the QSH edge states gap to yield six corner modes pinned to mirror lines and related by sixfold rotational symmetry. Since the corner modes are eigenstates of the chiral symmetry, they must be localized to a single sublattice. We can then include chiral symmetry breaking perturbatively to find that the corner modes are lifted from zero eigenvalue (or $\omega=\omega_{\text {LSP }}$ ), consistent with calculations as we discuss next.

We study the effect of LRIs by introducing an artificial cutoff in the CDM. We introduce an exponential decay to the dipole-dipole interactions, $f_{\text {c.o. }}\left(d_{i j}\right)=\exp \left[-\left(d_{i j}-\right.\right.$ $\left.d_{i j}^{0}\right) /\left(d_{i j}^{0} \gamma\right)$ ], where $d_{i j}^{0}$ is the nearest-neighbor separation for each dipole and $\gamma$ is a cut-off parameter to control the interaction range [38]. This allows us to continuously tune the interaction range from nearest neighbors $(\gamma=0.1)$, to electronic-like exponentially suppressed ones, all the way to full dipolar interactions $(\gamma \approx 5)$, as we show in Fig. 3(b) for $\delta=0.2$. For small values of $\gamma$, interactions in practice are only between nearest neighbours, such that there is no coupling between dipoles of the same sublattice. This preserves chiral symmetry and results in a spectrum that is symmetric about zero eigenvalue, with six degenerate topological corner modes (red) that are pinned at zero. Increasing the range of the interaction breaks chiral symmetry through coupling of elements in the same sublattice. This shifts the corner modes away from zero eigenvalue, lifts their de-
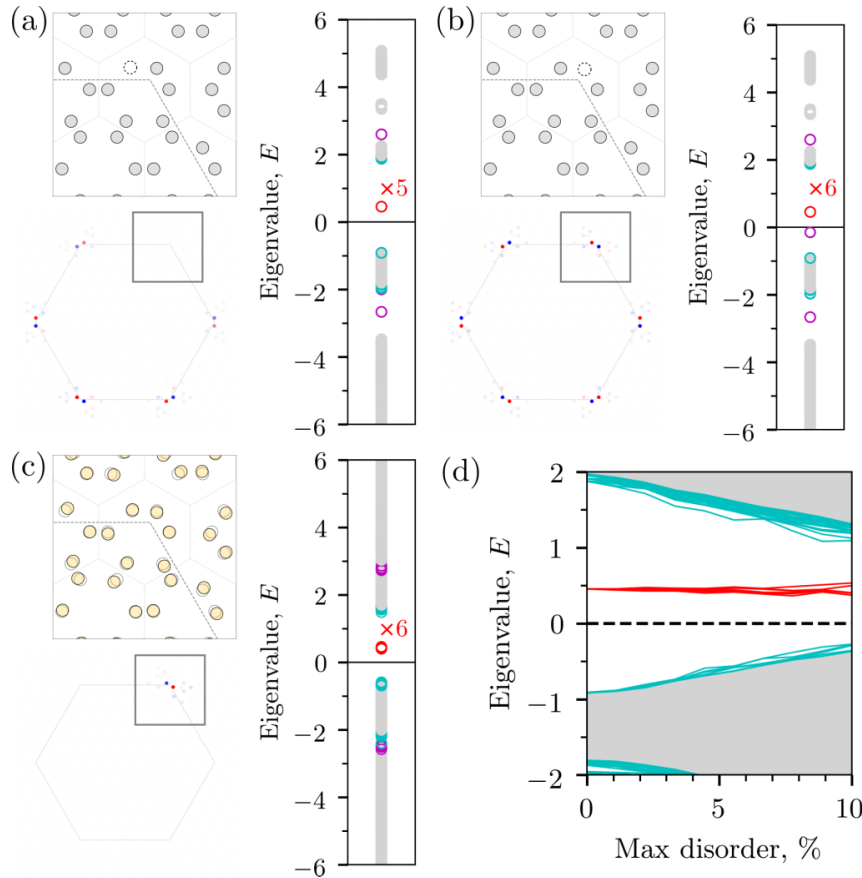

FIG. 4. Robustness of corner states against defects and disorder in the quasistatic model, for $\delta=0.2$. (a) A $C_{6}$-symmetry breaking defect in the lattice affects the midgap corner modes. (b) The corner states are robust against another kind of $C_{6}$-symmetry breaking defect due to the corner modes being close to chiral symmetric. (c) The degeneracy of the corner modes is lifted by random disorder: the position of the lattice sites is shifted randomly up to $5 \%$. (d) Eigenvalue spectrum for increasing positional disorder.

generacy (from six degenerate states to $1+2+2+1$, as in Fig. 2), and removes the symmetry of the spectrum about zero eigenvalue.

Robustness against defects and disorder. We now take advantage of the CDM to test the degree of protection of the corner modes against defects. We quantify protection by evaluating if the number of states within the band gap, together with the symmetries and degeneracies they satisfy, are left invariant. First, we create a strong defect in the crystal by removing one lattice site next to the corner of the particle, Fig. 4(a). Since this breaks the $C_{6}$ and mirror symmetries that protect the corner modes, one of them disappears and the remaining five satisfy new symmetry relations and degeneracies (see field plots and eigenvalue spectrum). Next, we consider removing one lattice site at exactly the corner Fig. 4(b), breaking the $C_{6}$ symmetry but respecting one mirror symmetry. Remarkably, the corner states are robust against this defect: there are six midgap states and they satisfy the same symmetries and degeneracies as before the perturbation. This is a consequence of the system being deformable to a chirally symmetric system. Despite the presence of LRIs, the modes still sit on alternating lattice sites, and the mode intensity is virtually zero at the removed lattice site. As a consequence, the $C_{6}$ symmetry of the mode is not affected by the removal of this lattice site, such that the $1+2+2+1$ degeneracy is mantained.

Finally, we test robustness against random positional disorder. In Fig. 4(c) we consider a system with maximum 5\% 
random disorder in lattice sites. Crucially, this breaks the $C_{6}$ symmetry across the whole lattice, such that the degeneracies of the corner modes are lifted, and each of the six midgap states localizes at one of the corners. On the other hand, we see in the spectrum how, despite the other corner modes and edge modes being lost to the bulk, the midgap corner modes remain well isolated at midgap energies. For practical purposes they are robust against random spatial perturbations. This is confirmed in Fig. 4(d), where we plot a closeup of the band gap and the HOTMs for increasing random positional disorder, up to a maximum of $10 \%$.

Conclusions. We have studied the emergence of topologically protected corner modes in breathing honeycomb $\mathrm{PhC}$ particles. By analyzing the lattice through topological quantum chemistry, Wilson loops, and the calculation of real-space topological invariants, we conclude that the topological properties emerge from an obstructed atomic limit phase, which in $2 \mathrm{D}$ is reminiscent of higher-order topology. Finally, we quantify the robustness of topological corner modes in $\mathrm{PhCs}$ to different kinds of perturbations. We conclude that, while LRIs inevitably break chiral symmetry, the corner modes are still protected by lattice symmetries. Although we have focused here on the breathing honeycomb lattice $\mathrm{PhC}$, our analysis applies to all classical wave systems.

Acknowledgments. M.P. and P.A.H. acknowledge funding from the Leverhulme Trust. P.A.H. acknowledges funding from Fundação para a Ciência e a Tecnologia and Instituto de Telecomunicações under projects CEECIND/03866/2017 and UID/50008/2020. B.B. acknowledges support of the Alfred P. Sloan Foundation and the National Science Foundation Grant No. DMR-1945058. A.G.E. and M.G.V. acknowledge support from DFG INCIEN2019-000356 from Gipuzkoako Foru Aldundia and the Spanish Ministerio de Ciencia e Innovacion (Grants No. PID2019-109905GB-C21 and No. PID2019-109905GA-C22). A.G.E. received funding from the Gipuzkoako Foru Aldundia OF23/2019 (ES) project and by Eusko Jaurlaritza Grants No. IT1164-19, No. PI2016-41, and No. KK-2019/00101. D.B. acknowledges support by the Spanish Ministerio de Ciencia, Innovation y Universidades (MICINN) through the project FIS201782804-P, and by the Transnational Common Laboratory Quantum-ChemPhys.
[1] T. Ozawa, H. M. Price, A. Amo, N. Goldman, M. Hafezi, L. Lu, M. C. Rechtsman, D. Schuster, J. Simon, O. Zilberberg, and I. Carusotto, Topological photonics, Rev. Mod. Phys. 91, 015006 (2019).

[2] L. Lu, J. D Joannopoulos, and M. Soljačić, Topological photonics, Nat. Photon. 8, 821 (2014).

[3] W. A. Benalcazar, B. A. Bernevig, and T. L. Hughes, Quantized electric multipole insulators, Science 357, 61 (2017).

[4] S. N. Kempkes, M. R. Slot, J. J. van den Broeke, P. Capiod, W. A. Benalcazar, D. Vanmaekelbergh, D. Bercioux, I. Swart, and C. Morais Smith, Robust zero-energy modes in an electronic higher-order topological insulator, Nat. Mater. 18, 1292 (2019).

[5] B. J. Wieder, Z. Wang, J. Cano, Xi Dai, L. M. Schoop, B. Bradlyn, and B A. Bernevig, Strong and fragile topological Dirac semimetals with higher-order Fermi arcs, Nat. Commun. 11, 627 (2020).

[6] W. A. Benalcazar, T. Li, and T. L. Hughes, Quantization of fractional corner charge in $C_{n}$-symmetric higher-order topological crystalline insulators, Phys. Rev. B 99, 245151 (2019).

[7] P. Zhu, K. Loehr, and T. L. Hughes, Identifying $C_{n}$-symmetric higher-order topology and fractional corner charge using entanglement spectra, Phys. Rev. B 101, 115140 (2020).

[8] C. W. Peterson, T. Li, W. A. Benalcazar, T. L. Hughes, and G. Bahl, A fractional corner anomaly reveals higher-order topology, Science 368, 1114 (2020).

[9] W. P. Su, J. R. Schrieffer, and A. J. Heeger, Solitons in Polyacetylene, Phys. Rev. Lett. 42, 1698 (1979).

[10] J. K. Asbóth, L. Oroszlány, and A. Pályi, A Short Course on Topological Insulators (Springer International Publishing, Cham, 2016).

[11] S. Imhof, C. Berger, F. Bayer, J. Brehm, L. W. Molenkamp, T. Kiessling, F. Schindler, C. H. Lee, M. Greiter, T. Neupert, and R. Thomale, Topolectrical-circuit realization of topological corner modes, Nat. Phys. 14, 925 (2018).

[12] Y. Ota, F. Liu, R. Katsumi, K. Watanabe, K. Wakabayashi, Y. Arakawa, and S. Iwamoto, Photonic crystal nanocavity based on a topological corner state, Optica 6, 786 (2019).

[13] B.-Y. Xie, G.-X. Su, H.-F. Wang, H. Su, X.-P. Shen, P. Zhan, M.-H. Lu, Z.-L. Wang, and Y.-F. Chen, Visualization of HigherOrder Topological Insulating Phases in Two-Dimensional Dielectric Photonic Crystals, Phys. Rev. Lett. 122, 233903 (2019).

[14] X.-D. Chen, W.-M. Deng, F.-L. Shi, F.-L. Zhao, M. Chen, and J.-W. Dong, Direct Observation of Corner States in SecondOrder Topological Photonic Crystal Slabs, Phys. Rev. Lett. 122, 233902 (2019).

[15] M. Li, D. Zhirihin, M. Gorlach, X. Ni, D. Filonov, A. Slobozhanyuk, A. Alù, and A. B. Khanikaev, Higher-order topological states in photonic kagome crystals with long-range interactions, Nat. Photon. 14, 89 (2020).

[16] J. Noh, W. A. Benalcazar, S. Huang, M. J. Collins, K. P. Chen, T. L. Hughes, and M. C. Rechtsman, Topological protection of photonic mid-gap defect modes, Nat. Photon. 12, 408 (2018).

[17] S. Mittal, V. V. Orre, G. Zhu, M. A. Gorlach, A. Poddubny, and M. Hafezi, Photonic quadrupole topological phases, Nat. Photon. 13, 692 (2019).

[18] A. El Hassan, F. K. Kunst, A. Moritz, G. Andler, E. J. Bergholtz, and M. Bourennane, Corner states of light in photonic waveguides, Nat. Photon. 13, 697 (2019).

[19] M. Serra-Garcia, V. Peri, R. Süsstrunk, O. R Bilal, T. Larsen, L. G. Villanueva, and S. D. Huber, Observation of a phononic quadrupole topological insulator, Nature (London) 555, 342 (2018). 
[20] X. Ni, M. A. Gorlach, A. Alu, and A. B. Khanikaev, Topological edge states in acoustic kagome lattices, New J. Phys. 19, 055002 (2017).

[21] X. Ni, M. Weiner, A. Alù, and A. B. Khanikaev, Observation of higher-order topological acoustic states protected by generalized chiral symmetry, Nat. Mater. 18, 113 (2019).

[22] Y. Qi, C. Qiu, M. Xiao, H. He, M. Ke, and Z. Liu, Acoustic Realization of Quadrupole Topological Insulators, Phys. Rev. Lett. 124, 206601 (2020).

[23] H. Fan, B. Xia, L. Tong, S. Zheng, and D. Yu, Elastic Higher-Order Topological Insulator with Topologically Protected Corner States, Phys. Rev. Lett. 122, 204301 (2019).

[24] C. W. Peterson, W. A. Benalcazar, T. L. Hughes, and G. Bahl, A quantized microwave quadrupole insulator with topologically protected corner states, Nature (London) 555, 346 (2018).

[25] M. Kim, Z. Jacob, and J. Rho, Recent advances in 2D, 3D and higher-order topological photonics, Light: Sci. Appl. 9, 130 (2020).

[26] H.-R. Kim, M.-S. Hwang, D. Smirnova, K.-Y. Jeong, Y. Kivshar, and H.-G. Park, Multipolar lasing modes from topological corner states, Nat. Commun. 11, 5758 (2020).

[27] C. Han, M. Kang, and H. Jeon, Lasing at multidimensional topological states in a two-dimensional photonic crystal structure, ACS Photon. 7, 2027 (2020).

[28] Y. Gong, S. Wong, A. J. Bennett, D. L. Huffaker, and S. S. Oh, Topological insulator laser using valley-Hall photonic crystals, ACS Photon. 7, 2089 (2020).

[29] A. F. Koenderink and A. Polman, Complex response and polariton-like dispersion splitting in periodic metal nanoparticle chains, Phys. Rev. B 74, 033402 (2006).

[30] S. R. Pocock, X. Xiao, P. A. Huidobro, and V. Giannini, Topological plasmonic chain with retardation and radiative effects, ACS Photon. 5, 2271 (2018).

[31] S. R. Pocock, P. A. Huidobro, and V. Giannini, Bulk-edge correspondence and long-range hopping in the topological plasmonic chain, Nanophotonics 8, 1337 (2019).

[32] L. He, Z. Addison, E. J. Mele, and B. Zhen, Quadrupole topological photonic crystals, Nat. Commun. 11, 3119 (2020).

[33] M. Jung, R. G. Gladstone, and G. B. Shvets, Nanopolaritonic second-order topological insulator based on graphene plasmons, Adv. Photon. 2, 046003 (2020).

[34] L.-H. Wu and X. Hu, Scheme for Achieving a Topological Photonic Crystal by Using Dielectric Material, Phys. Rev. Lett. 114, 223901 (2015).

[35] B. Xie, G. Su, H.-F. Wang, F. Liu, L. Hu, S.-Y. Yu, P. Zhan, M.-H. Lu, Z. Wang, and Y.-F. Chen, Higher-order quantum spin Hall effect in a photonic crystal, Nat. Commun. 11, 3768 (2020).

[36] F. J. García de Abajo, Colloquium: Light scattering by particle and hole arrays, Rev. Mod. Phys. 79, 1267 (2007).

[37] F. Liu, H.-Y. Deng, and K. Wakabayashi, Helical Topological Edge States in a Quadrupole Phase, Phys. Rev. Lett. 122, 086804 (2019).

[38] See Supplemental Material at http://link.aps.org/supplemental/ 10.1103/PhysRevResearch.2.042038 for bulk band structures and Wilson loops of both phases, topological analysis of the quasistatic analytical model, details on the coupled dipole model and exponential cut-off, and a summary table on defects and disorder.
[39] S. Barik, A. Karasahin, C. Flower, T. Cai, H. Miyake, W. DeGottardi, M. Hafezi, and E. Waks, A topological quantum optics interface, Science 359, 666 (2018).

[40] M. A. Gorlach, X. Ni, D. A. Smirnova, D. Korobkin, D. Zhirihin, A. P. Slobozhanyuk, P. A. Belov, A. Alù, and A. B. Khanikaev, Far-field probing of leaky topological states in alldielectric metasurfaces, Nat. Commun. 9, 909 (2018).

[41] S. Peng, N. J. Schilder, X. Ni, J. van de Groep, M. L. Brongersma, A. Alù, A. B. Khanikaev, H. A. Atwater, and A. Polman, Probing the Band Structure of Topological Silicon Photonic Lattices in the Visible Spectrum, Phys. Rev. Lett. 122, 117401 (2019).

[42] D. Smirnova, S. Kruk, D. Leykam, E. Melik-Gaykazyan, D.-Y. Choi, and Y. Kivshar, Third-Harmonic Generation in Photonic Topological Metasurfaces, Phys. Rev. Lett. 123, 103901 (2019).

[43] N. Parappurath, F. Alpeggiani, L. Kuipers, and E. Verhagen, Direct observation of topological edge states in silicon photonic crystals: Spin, dispersion, and chiral routing, Sci. Adv. 6, eaaw4137 (2020).

[44] W. Liu, M. Hwang, Z. Ji, Y. Wang, G. Modi, and R. Agarwal, $z_{2}$ photonic topological insulators in the visible wavelength range for robust nanoscale photonics, Nano Lett. 20, 1329 (2020).

[45] Z.-Q. Yang, Z.-K. Shao, H.-Z. Chen, X.-R. Mao, and R.-M. Ma, Spin-Momentum-Locked Edge Mode for Topological Vortex Lasing, Phys. Rev. Lett. 125, 013903 (2020).

[46] J. Cano, B. Bradlyn, Z. Wang, L. Elcoro, M. G. Vergniory, C. Felser, M. I. Aroyo, and B. A. Bernevig, opology of Disconnected Elementary Band Representations, Phys. Rev. Lett. 120, 266401 (2018).

[47] B. Bradlyn, L. Elcoro, J. Cano, M. G. Vergniory, Z. Wang, C. Felser, M. I. Aroyo, and B. A. Bernevig, Topological quantum chemistry, Nature (London) 547, 298 (2017).

[48] M. B. de Paz, M. G. Vergniory, D. Bercioux, A. García-Etxarri, and B. Bradlyn, Engineering fragile topology in photonic crystals: Topological quantum chemistry of light, Phys. Rev. Res. 1, 032005 (2019).

[49] R. M. Geilhufe and W. Hergert, GTPack: A Mathematica group theory package for application in solid-state physics and photonics, Front. Phys. 6, 86 (2018).

[50] W. Hergert and R. M. Geilhufe, Group Theory in Solid State Physics and Photonics: Problem Solving with Mathematica (Wiley-VCH, Weinheim, 2018).

[51] M. I. Aroyo, J. M. Perez-Mato, D. Orobengoa, E. M. R. E. Tasci, G. de la Flor, and A. Kirov, Crystallography online: Bilbao crystallographic server, Bulg. Chem. Commun. 43, 183 (2011).

[52] M. I. Aroyo, J. M. Perez-Mato, C. Capillas, E. Kroumova, S. Ivantchev, G. Madariaga, A. Kirov, and H. Wondratschek, Bilbao crystallographic server: I. Databases and crystallographic computing programs, Z. Krist.-Crystal. Mater. 221, 15 (2006).

[53] M. I. Aroyo, A. Kirov, C. Capillas, J. M. Perez-Mato, and H. Wondratschek, Bilbao crystallographic server. II. Representations of crystallographic point groups and space groups, Acta Cryst. A62, 115 (2006).

[54] M. G. Vergniory, L. Elcoro, Z. Wang, J. Cano, C. Felser, M. I. Aroyo, B. A. Bernevig, and B. Bradlyn, Graph theory data for topological quantum chemistry, Phys. Rev. E 96, 023310 (2017).

[55] L. Elcoro, B. Bradlyn, Z. Wang, M. G. Vergniory, J. Cano, C. Felser, B. A. Bernevig, D. Orobengoa, G. de la Flor, and 
M. I. Aroyo, Double crystallographic groups and their representations on the Bilbao crystallographic server, J. Appl. Cryst. 50, 1457 (2017).

[56] We note that we have characterized the topology of the system using bands $4-6$, in order to avoid cusp at $\omega=k=0$. Whilst there may be additional topological features in the lower bands here [68], they do not affect the corner mode analysis in this Rapid Communication.

[57] M. Blanco de Paz, C. Devescovi, G. Giedke, J. J. Saenz, M. G. Vergniory, B. Bradlyn, D. Bercioux, and A. GarcíaEtxarri, Tutorial: Computing topological invariants in 2D photonic crystals, Adv. Quantum Technol. 3, 1900117 (2020).

[58] D. Vanderbilt, Berry Phases in Electronic Structure Theory: Electric Polarization, Orbital Magnetization and Topological Insulators (Cambridge University Press, Cambridge, UK, 2018).

[59] In the following, chiral (sublattice) symmetry refers to the Hamiltonian $H(k)$ of a system satisfying $\Gamma^{-1} H(k) \Gamma=-H(k)$ [10]. The chiral symmetry operator $\Gamma$ takes the form $\sigma_{z} \otimes \mathbb{I}$, where $\sigma_{z}$ is the Pauli spin matrix.

[60] M. Ezawa, Higher-Order Topological Insulators and Semimetals on the Breathing Kagome and Pyrochlore Lattices, Phys. Rev. Lett. 120, 026801 (2018).
[61] Z.-D. Song, L. Elcoro, and B. A. Bernevig, Twisted bulkboundary correspondence of fragile topology, Science 367, 794 (2020).

[62] G. van Miert and C. Ortix, Higher-order topological insulators protected by inversion and rotoinversion symmetries, Phys. Rev. B 98, 081110(R) (2018).

[63] G. van Miert and C. Ortix, On the topological immunity of corner states in two-dimensional crystalline insulators, npj Quantum Mater. 5, 63 (2020).

[64] G. Siroki, P. A. Huidobro, and V. Giannini, Topological photonics: From crystals to particles, Phys. Rev. B 96, 041408(R) (2017).

[65] M. J. Mehrabad, A. P. Foster, R. Dost, E. Clarke, P. K. Patil, I. Farrer, J. Heffernan, M. S. Skolnick, and L. R. Wilson, A semiconductor topological photonic ring resonator, Appl. Phys. Lett. 116, 061102 (2020).

[66] S. Barik, A. Karasahin, S. Mittal, E. Waks, and M. Hafezi, Chiral quantum optics using a topological resonator, Phys. Rev. B 101, 205303 (2020).

[67] S. G. Johnson and J. D. Joannopoulos, Block-iterative frequency-domain methods for Maxwell's equations in a planewave basis, Opt. Express 8, 173 (2001).

[68] H. Watanabe and L. Lu, Space Group Theory of Photonic Bands, Phys. Rev. Lett. 121, 263903 (2018). 